RESEARCH

\title{
OSTEOPOROSIS AND HIP-FRACTURE RISK ASSESSMENT USING DIFFERENT TOOLS IN THE FIRST YEAR AFTER STROKE
}

Turkish Journal of Geriatrics

DOI: 10.31086/tigeri.2021.209

2021; 24(2): 143-149

- Nurdan PAKER ${ }^{1}$

- Derya BUĞDAYCI ${ }^{1}$

- Halime KIBAR ${ }^{2}$

- Yelda SOLUK ÖZDEMIR ${ }^{1}$

CORRESPONDANCE

${ }^{1}$ Nurdan PAKER

Istanbul Physical Medicine and Rehabilitation

Training and Research Hospital, Physical

Medicine and Rehabilitation, istanbul, Turkey

Phone: +905326917591

e-mail: nurdanpaker@hotmail.com

Received: Dec 12, 2020

Accepted: May 02, 2021

1 Istanbul Physical Medicine and Rehabilitation Training and Research Hospital, Physical Medicine and Rehabilitation, istanbul, Turkey

2 Bağcllar Training and Research Hospital, Physical Medicine and Rehabilitation, İstanbul, Turkey

\section{Abstract}

Purpose: The aim of this study was to ascertain the frequency of osteoporosis and 10-year hip-fracture risk in patients with post-stroke hemiplegia using two different assessment algorithms.

Method: Seventy-seven patients with post-stroke hemiplegia $\leq 1$ year who were admitted to the stroke unit of a tertiary rehabilitation hospital were included in this cross-sectional study. All patients underwent bone density assessment. Fracture risk assessment tool (FRAX) and QFracture were both used to calculate the 10-year risk probability for hip fracture. Receiver operating characteristic curves were investigated for FRAX and QFracture.

Results: Fifteen patients (19.5\%) had osteoporosis, and 39 (50.6\%) had osteopenia based on dual-energy X-ray absorptiometry measurements. FRAX hip scores were $\geq 3$ in 7 patients (9.1\%). Ten-year hip-fracture risk was high in 26 patients (33.8\%) using QFracture. Six patients (7.8\%) had low-energy fractures after stroke. Fracture sites were hip in 2 patients, 1 humerus, 1 forearm, and 2 in lower extremities. The receiver operating characteristic area under curve was 0.804 (\% $95 \mathrm{Cl}$ 0.665-0.943, $\mathrm{p}=0.002$ ) for FRAX and 0.758 (\% $95 \mathrm{Cl}$ 0.615-0.901, $p=0.009)$ for QFracture. Cut-off values for the estimation of hip osteoporosis for FRAX and QFracture tools were 1.15 and 1.55, respectively.

Conclusion: Findings indicated that $70.1 \%$ of patients in this study had osteoporosis or osteopenia within the first year following a stroke. Ten-year hip-fracture risk was $9.1 \%$ by FRAX and $33.8 \%$ by QFracture. Results of FRAX and QFracture assessment tools demonstrated a significant relationship between ten-year hip-fracture risk and bone mineral density.

Keywords: Stroke; Hemiplegia; Osteoporosis; Bone Density 


\section{INTRODUCTION}

Fracture risk increases after stroke due to decreased bone density and high fall tendency (1-3). Patients with stroke have 1.4-2.4-fold increased hip fracture risk (4). Advanced age, female gender, a history of previous fractures or falls, having diseases that affect bone metabolism, diminished mental status, and atrial fibrillation may all be risk factors for hip fracture in stroke patients $(2,3,5)$.

Although the fracture risk is high in people with hemiplegia, there are no standardized screening tests. Fracture risk assessment in hemiplegic patients is essential prophylactic prevention of fractures and in identifying the individuals who need osteoporosis treatment. Bone mineral density (BMD) assessment is helpful in recognizing patients with osteoporosis and determining their fracture risk. Usually, osteoporosis treatment is advised for patients with BMD T-scores $\leq-2.5$ at lumbar spine and/or femoral neck. People with BMD T-scores between -1 and -2.5 are diagnosed as having osteopenia and can be treated for increased fracture risk. BMD measurement can be performed after vital signs are stable in clinical practice, especially for stroke patients at high risk of osteoporosis. Dual-energy X-ray absorptiometry (DXA) measurement rate is very low among stroke patients (6). Stroke is a serious health problem with various complications other than osteoporosis (7). For this reason, importance of screening for osteoporosis and assessing high fracture risk may be overlooked.

Fracture Risk Assessment Tool (FRAX) and QFracture are absolute fracture risk prediction algorithms $(8,9)$. However, the efficacy of these tests in determining hip-fracture risk in stroke patients has not been clearly established. The FRAX tool helps identify individuals with high fracture risk associated with osteoporosis and in determining the need for treatment. FRAX is considered superior to BMD measurement alone in treatment decision-making (8). Twelve items are examined with FRAX, including the presence of secondary osteoporosis. In fact, there are more diseases that may lead to secondary osteoporosis. No item in FRAX deals with falls that may have been influenced by medical treatment. Stroke may lead to secondary osteoporosis due to immobilization and hemiplegia but it is not presented as a cause of secondary osteoporosis in FRAX.

The QFracture tool was developed in England to calculate the risk of osteoporotic fracture including hip fractures. QFracture was especially promoted for determining both absolute osteoporotic and hip-fracture risks in the primary care setting. The fracture risk calculation with QFracture can be performed in two ways. First, fracture risk is assessed by the clinician according to existing electronic records. Second, the items can be completed by the patient because the test does not involve laboratory or other empirical testing. Items such as fragility, fracture history, etnic group, epilepsy, and the use of antidepressants, living in a nursing home, inflammatory arthropathies, chronic obstructive pulmonary disease (COPD), type I diabetes mellitus, Parkinson's disease, and dementia leading to immobilization were added to this algorithm in 2012 (9). Both FRAX and QFracture tools can be used to predict hip-fracture risk (10-12), but there is no algorithm specific to stroke for identifying patients who need osteoporosis treatment.

The aim of this study was to assess the rate of osteoporosis and to compare the efficacy of FRAX and QFracture algorithms in identifying patients with high hip-fracture risk at one year after stroke.

\section{METHODS}

Hundred patients with hemiplegia admitted to the inpatient stroke rehabilitation unit between 01.03.2016-30.11.2016 were screened in this cross-sectional study. Stroke diagnosis was made clinically and verified by magnetic resonance imaging studies. Exclusion criteria were age under 40 years, post-stroke duration $>12$ months, a history of osteoporosis, use of antiresorptive drugs, and hav- 
ing a condition affecting bone metabolism. One patient (28 years) was excluded due to very young age. In addition, fourteen patients whose disease durations were $>12$ months and eight patients who had a previous diagnosis of osteoporosis were excluded from the study. Seventy-seven patients with hemiplegia were enrolled. Disability was assessed by the Modified Rankin Scale which is a valid and reliable test for stroke patients (13). It has six stages: Grade 1 indicates the patients without significant disability, whereas grade 5 indicates severe disability. Grade 6 denotes death. Ambulation status was recorded as non-ambulatory, therapeutic ambulation, household ambulation, and functional ambulation.

Written informed consent was obtained from all patients. The study was approved by the hospital ethical committee.

\section{Bone density measurement}

Lumbar region and non-hemiplegic femur neck BMD values were measured using dual-energy X-ray absorptiometry (Lunar Dpx, Pro) and T-scores were recorded. Non-hemiplegic hip bone density values were used in this study. The hemiplegic proximal femur bone density measurement may not be feasible due to factors such as previous fracture or hip prosthesis, heterotopic ossification, and improper positioning of the leg caused by spasticity or contractures. T-score is the standard deviation of the patient's BMD measurement scores from those of a young healthy adult. Lumbar region and/or femur neck T-scores $\leq-2.5$ indicate osteoporosis, and T-scores between -2.5 and -1 are diagnosed as osteopenia. Low BMD values indicate a higher fracture risk (14).

\section{Fracture Risk Assessment Tool (FRAX)}

FRAX is recognized by the World Health Organization for estimation of ten-year major osteoporotic or hip-fracture risks (15). FRAX is an easy web-based tool that helps estimate the probability of ten-year fracture risk as a percentage (16). Age, sex, race, height, weight, body mass index, previous fragility fracture, family hip-fracture history, treatment with oral glucocorticoids, rheumatoid arthritis, other conditions that may cause secondary osteoporosis, smoking, and daily alcohol intake are inquired in the FRAX. It can be used with or without femur neck $B M D$ values. FRAX without BMD is usually accepted as adequate for making treatment decisions $(15,17)$. In this study, FRAX without BMD was used to compare the results of different tools for the patients who have no access to DXA measurement.

\section{QFracture}

The QFracture tool is used to predict the tenyear hip or osteoporotic fracture risk in clinical settings, including primary care. It can also be used as a web-based self-assessment tool (18). BMD values and other laboratory tests are not required for calculating the score. QFracture was updated in 2012 (9). Risk factors questioned in QFracture are age, sex, ethnicity, body mass index, weight, height, smoking, alcohol use, previous fractures or family history of fractures, hormone replacement therapy, menopausal symptoms, endocrine problems, treatment with glucocorticoids, disorders that lead to secondary osteoporosis (type I diabetes, chronic hyperthyroidism, premature menopause, chronic malnutrition, chronic liver disease), asthma, cardiovascular disease (heart attack, angina, stroke, TIA), history of falls, malabsorption, Parkinson's disease, rheumatoid arthritis, SLE, type 2 diabetes, epilepsy or use of anticonvulsants, and treatment with antidepressants. Stroke is appraised as a separate risk factor in QFracture. Completing the QFracture is more time-consuming than the FRAX. QFracture scores are expressed as a percentage for both 10year osteoporotic and hip-fracture risks. It has been shown that a cut-off value of $5.3 \%$ would identify 3295 (59.8\%) of the 5509 women with a hip fracture over the next 10 years. Similarly in men the cutoff value for hip fracture was determined as 1.3\% with $64.3 \%$ sensitivity. Cut-off values are adapted from 10-year predicted risk for hip fracture in the validation cohort by Hippisley-Cox J (9). QFracture 
has been developed in England, however, the validation of QFracture for the other countries has not been done (19).

\section{STATISTICAL ANALYSIS}

The suitability of the data was evaluated with the Shapiro-Wilk test and homogeneity was evaluated with the Levene test. Scale data were shown as arithmetic mean \pm standard deviation or ordinal data were given as median (minimum-maximum) and categorical data were expressed as $\mathrm{n}(\%)$. The receiver operating characteristic $(\mathrm{ROC})$ curves were evaluated for several variables based on the cut-off value that maximizes both sensitivity and specificity of the tools. Test variables were FRAX without BMD and OFracture; the state variable was the presence of osteoporosis according to T-scores of femoral neck and lumbar spine. The ROC curve was constructed, and the area under curve (AUC) and its $95 \%$ confidence interval $(\mathrm{Cl})$ were estimated. A $p$ value $\leq 0.05$ was considered statistically significant. The SPSS 22.0 (IBM Corporation) program was used for variable analyses.

\section{RESULTS}

Demographic features and clinical characteristics are summarized in Tables 1 and 2. Fifteen patients (19.5\%) had osteoporosis and 39 (50.6\%) had osteopenia. Non-hemiplegic hip T-scores were $\leq-2.5$ in 10 patients (13.0\%) and between -1 and -2.5 in 35 patients (45.5\%). Mean FRAX 10-year hip-fracture score was $1.4 \pm 2.0$. Seven patients (9.1\%) had FRAX hip scores $\geq 3$. Mean QFracture score for hip fracture was $2.5 \pm 3.5$. Ten-year hip-fracture risk was high in 26 patients (33.8\%) using QFracture. Six patients $(7.8 \%)$ had low-energy fractures after stroke. Fracture sites were hip in 2 patients, 1 humerus, 1 forearm, and 2 in lower extremities.

The ROC curves of FRAX without BMD and QFracture for the risk of osteoporosis are shown in Figure 1. All variables were found valid for predict-
Table 1. Demographic characteristics

\begin{tabular}{|l|c|}
\hline Age (yrs) & $62.2 \pm 11.1$ \\
\hline Female/Male (,$\%)$ & $27 / 50(35.1 / 64.9 \%)$ \\
\hline Married/Single (n, \%) & $56 / 21(72.3 / 27.3 \%)$ \\
\hline BMl (kg/m²) & $26.9 \pm 4.4$ \\
\hline Time since stroke (mos) & $3.9 \pm 2.6$ \\
\hline Right/left hemiplegia (n, \%) & $38 / 39(49.4 / 50.6 \%)$ \\
\hline Ischemic/haemorrhagic $(n, \%)$ & $67 / 10(87.0 / 13.0 \%)$ \\
\hline
\end{tabular}

Data were given as the arithmetic mean \pm standard deviation or number (percent)

ing risk of hip osteoporosis. The area under the $\mathrm{ROC}$ curve was 0.804 (\% $95 \mathrm{Cl} 0.665-0.943, \mathrm{p}=0.002)$ for FRAX and 0.758 (\% $95 \mathrm{Cl} 0.615-0.901, \mathrm{p}=0.009$ ) for QFracture. Cut-off values for the estimation of hip osteoporosis for FRAX and QFracture tools were 1.15 and 1.55 , respectively (Table 3).

Table 2. Clinical characteristics

\begin{tabular}{|l|c|}
\hline Brunnstrom stage Upper Extremity & $2(1-6)$ \\
\hline Brunnstrom Stage Lower Extremity & $3(1-6)$ \\
\hline Modified rankin scores & $3(1-5)$ \\
\hline Ambulation status & $3(1-5)$ \\
\hline FRAX score & $1.4 \pm 2.0$ \\
\hline QFracture score & $2.5 \pm 3.5$ \\
\hline Intact femur neck BMD $\left(\mathrm{g} / \mathrm{cm}^{2}\right)$ & $0.903 \pm 0.156$ \\
\hline Intact femur neck T-score & $-1.1 \pm 1.1$ \\
\hline
\end{tabular}

Data were given as the median (min-max) or arithmetic mean \pm standard deviation 
Figure 1. Area under curve of FRAX and QFracture for hip osteoporosis

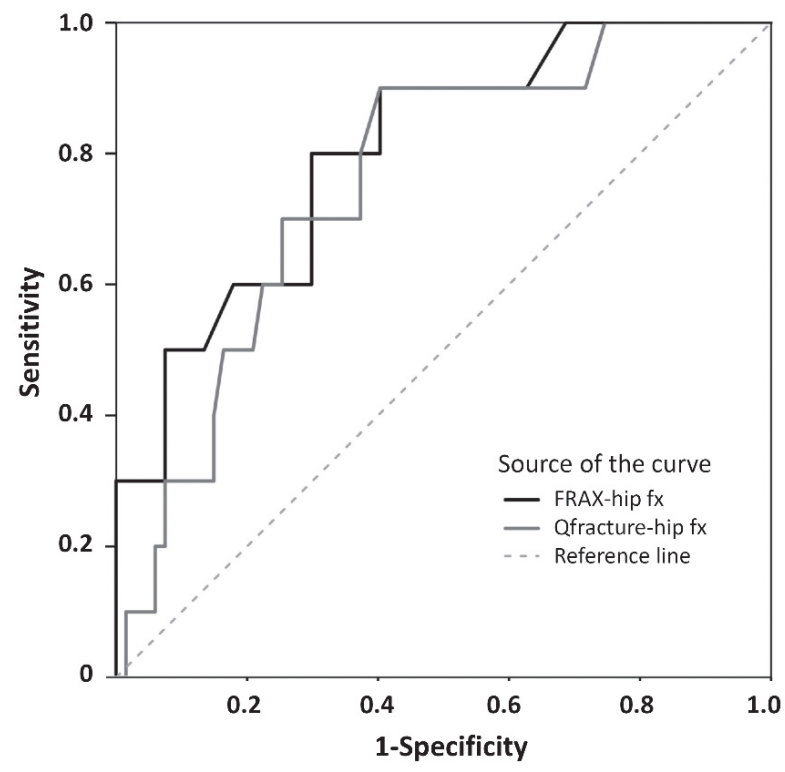

\section{DISCUSSION}

In this study, $19.5 \%$ of the patients with hemiplegia $\leq 1$ year post-stroke had osteoporosis and 50.6\% had osteopenia. The osteoporosis risk increased 1.77-fold after stroke (20). However, routine DXA scanning may not be possible in patients with hemiplegia. In a recent study, the bone density measurement rate was reported as low as $5.1 \%$ at one year after stroke (6). Moreover, the authors concluded that only $15.5 \%$ had medical treatment for osteoporosis. DXA measurement is usually not possible in the acute phase following stroke due to many systemic problems. After discharge, access to DXA is usually not easy because of transportation difficulty of the disabled person or accompanying medical conditions. Thus, fracture risk assessment tools can help identify patients who need treatment to prevent low-energy trauma fractures.

An interesting finding of this study is that $9.1 \%$ and $33.8 \%$ of the patients had increased 10 -year hip-fracture risk according to the FRAX and QFracture tools, respectively. Fall history and neurological diseases including stroke are inquired in the QFracture tool; however, FRAX does not contain any stroke-related items. In a study conducted with Parkinson's disease patients, it was found that the fracture risk is mildly lower as assessed by FRAX compared to evaluations by QFracture (21). The rate of stroke patients having high 10-year hip-fracture risk was lower by FRAX in this study, in parallel with findings in previous research.

10-year hip fracture risk calculated with OFracture was found significantly higher that the risk calculated with FRAX among women in an osteoporosis outpatient clinic in a previously (22). FRAX and QFracture results are found correlated in the patients with a history of low-trauma hip fracture or injury risk in another study (12). On the other hand, the authors concluded that FRAX is less effective in determining hip-fracture risk. QFracture was found to perform better in identifying patients with a history of falls and determining the treatment threshold. QFracture and FRAX yielded high discriminato-

Table 3. Comparison between cut-off values, sensitivities, and specificities of the tools involved in osteoporosis risk assessment

\begin{tabular}{|c|c|c|c|c|c|c|c|}
\hline $\begin{array}{c}\text { State } \\
\text { Variable }\end{array}$ & $\begin{array}{c}\text { Test Result } \\
\text { Variables }\end{array}$ & AUC $(\mathbf{9 5 \%} \mathrm{Cl})$ & P value & $\begin{array}{c}\text { Cut-off } \\
\text { value }\end{array}$ & $\begin{array}{c}\text { Jouden } \\
\text { index }\end{array}$ & Sensitivity & Specificity \\
\hline Hip OP & FRAX-hip fx & $0.804(0.665-0.943)$ & 0.002 & 1.150 & 0.501 & 0.800 & 0.701 \\
\hline Hip OP & QFracture-hip fx & $0.758(0.615-0.901)$ & 0.009 & 1.550 & 0.497 & 0.900 & 0.597 \\
\hline
\end{tabular}

AUC: area under curve, OP osteoporosis, $\mathrm{Cl}$ : confidence interval, fx: fracture 
ry performance for hip fracture prediction, however, QFracture is thought to be slightly better in a retrospective cohort study with more that 1 million individuals between ages 50-90 (11).

In our study, there was a correlation between FRAX and QFracture results. FRAX scores were significantly high in patients with previous hip and/ or peripheral fractures. In addition, among the patients with or without fracture history, no significant difference was detected in terms of QFracture scores. Because of the limited number of the patients with previous fractures, we cannot claim one tool is better than the other.

In this study, six patients (7.8\%) had low-energy fractures with a mean disease duration of 3 months. Fracture sites were hip and lower and upper extremities. The hip fracture rate was $2.6 \%$. Lower extremity fractures are common after stroke (23). In a population-based study, 2-year low-trauma fracture frequency was reported to be $5.7 \%$, whereas femur fracture rate was $2.8 \%$ in stroke (5).

According to the ROC curves, both osteoporotic fracture risk estimation tools were found satisfactory as compared with BMD values. The predictive value of the tools was determined according to the area under the ROC curve (AUC) as follows: non-predictive (AUC < 0.5), less predictive (AUC: 0.5-0.7), moderately predictive (AUC: 0.7-0.9), highly predictive (AUC: $0.9-1)$, and perfectly predictive $(A \cup C=1)$ $(24,25)$. If the FRAX hip-fracture score is $>1.2 \%$, os-

\section{REFERENCES}

1. Luan L, Li R, Wang Z, et al. Stroke increases the risk of hip fracture: a systematic review and meta-analysis. Osteoporos Int 2016;27(11):3149-3154. (PMID: 27185402)

2. Yuan ZC, Mo H, Guan J, et al. Risk of hip fracture following stroke, a meta-analysis of 13 cohort studies. Osteoporos Int 2016;27(9):2673-2679. (PMID: 27101998) teoporosis can be predicted at the plegic hip with a sensitivity of $75 \%$. When the QFracture hip-fracture score is $>4.8 \%$, osteoporosis can be predicted at the plegic hip with a sensitivity of $75 \%$. Some screening tools, including FRAX, were previously reported to be reliable for estimating osteoporosis in postmenopausal women, in line with our results (26).

The main strength of our study is, to our knowledge, it is the first report that compares FRAX and QFracture tools in patients with hemiplegia. A limitation is that QFracture was developed for England, and it has not been validated in Turkey, yet. Lacking long-term follow-up due to its cross-sectional design is another limitation. There were no patients with osteoporosis diagnosis before stroke but not all of them had DXA measurement previously. Moreover, the results cannot be generalized to all stroke patients, because this study included patients with hemiplegia who were admitted to a tertiary rehabilitation hospital.

In conclusion, almost three-fourths of patients have osteoporosis or osteopenia within one year following stroke in this study. QFracture may be better for the hip fracture risk assessment because of the items related with stroke, although it is not validated epidemiologically in Turkey. Stroke-specific fracture tools are needed in performing risk assessment for patients who cannot access BMD measurement.

3. Dennis MS, Lo KM, McDowall M, West T. Fractures after stroke: frequency, types, and associations. Stroke. 2002;33(3):728-34. (PMID: 11872896)

4. Lin $H L$, Lin $H C$, Tseng $Y F$, et al. Hip fracture after first-ever stroke: a population-based study. Acta Neurol Scand. 2015 ;131(3):158-63. (PMID: 25263230)

5. Kapral MK, Fang J, Alibhai SM, et al. Risk of fractures after stroke: Results from the Ontario Stroke Registry. Neurology. 2017;88(1):57-64. (PMID: 27881629) 
6. Kapoor E, Austin PC, Alibhai SMH, et al. Screening and treatment for osteoporosis after stroke. Stroke. 2019;50(6):1564-6. (PMID: 31018778)

7. Winstein CJ, Stein J, Arena R, et al. Guidelines for adult stroke rehabilitation and recovery: a guideline for healthcare professionals from the american heart association/american stroke association. Stroke. 2016; 47:e98-e169. (PMID: 27145936)

8. Kanis JA, Hans D, Cooper C, et al. Task Force of the FRAX Initiative. Interpretation and use of FRAX in clinical practice. Osteoporos Int 2011;22:2395-411. (PMID: 21779818)

9. Hippisley-Cox J, Coupland C. Derivation and validation of updated QFracture algorithm to predict risk of osteoporotic fracture in primary care in the United Kingdom: prospective open cohort study BMJ 2012;344:e3427. (PMID: 22619194)

10. Kanis JA, Compston J, Cooper C et al. SIGN Guidelines for Scotland: BMD Versus FRAX Versus QFracture. Calcif Tissue Int 2016;98:417-25. (PMID: 26650822)

11. Dagan N, Cohen-Stavi C, Leventer-Roberts M, Balicer RD. External validation and comparison of three prediction tools for risk of osteoporotic fractures using data from population based electronic health records: retrospective cohort study. BMJ. 2017;356:i6755. (PMID: 28104610)

12. Johansen A. QFracture is better than FRAX tool in assessing risk of hip fracture. BMJ. 2012;345:4988. (PMID: 22826597)

13. Banks JL, Marotta CA. Outcomes validity and reliability of the modified Rankin scale: implications for stroke clinical trials: a literature review and synthesis. Stroke. 2007;38(3):1091-6. (PMID: 17272767)

14. Leslie WD, Tsang JF, Caetano PA, Lix LM. Bone Density Program. Effectiveness of bone density measurement for predicting osteoporotic fractures in clinical practice. J Clin Endocrinol Metab. 2007;92(1):77-81. (PMID: 17032716)

15. Gadam RK, Schlauch K, Izuora KE. FRAX prediction without BMD for assessment of osteoporotic fracture risk. Endocr Pract. 2013;19(5):780-784. (PMID: 24121261)

16. Kanis JA, Oden A, Johnell $O$, et al. The use of clinical risk factors enhances the performance of BMD in the prediction of hip and osteoporotic fractures in men and women. Osteoporos Int. 2007 ;18(8):1033-46. (PMID: 17323110)

17. Simpkins RC, Downs TN, Lane MT. FRAX prediction with and without bone mineral density testing. Fed Pract. 2017;34(5):40-43. (PMID: 30766280)

18. Hippisley-Cox J, Coupland C. Predicting risk of osteoporotic fracture in men and women in England and Wales: prospective derivation and validation of OFracture Scores. BMJ. 2009;339:b4229. (PMID: 19926696)

19. Kanis JA, Harvey NC, Johansson H, Odén A, McCloskey EV, Leslie WD. Overview of Fracture Prediction Tools. J Clin Densitom. 2017 ;20(3):444-450.

20. Zhang L, Zhang ZH, Wang QR, et al. Stroke and osteoporosis: a Taiwan cohort study. Postgrad Med J. 2020 Mar 12:postgradmedj-2019-136959. (PMID: 32165547)

21. Lyell V, Henderson E, Devine M, Gregson C. Assessment and management of fracture risk in patients with Parkinson's disease. Age Ageing 2015;44:34-41. (PMID: 25236847)

22. Aw D, Thain J, Ali A, Aung T, Chua WM, Sahota O, Weerasuriya N, Marshall L, Kearney F, Masud T. Predicting fracture risk in osteoporosis: the use of fracture prediction tools in an osteoporosis clinic population. Postgrad Med J. 2016 May;92(1087):267-70. doi: 10.1136/postgradmedj-2015-133454. PMID: 26792635.

23. Huo K, Hashim SI, Yong KL, Su H, Qu QM. Impact and risk factors of post-stroke bone fracture. World J Exp Med. 2016;6(1):1-8. (PMID: 26929915)

24. Greiner M, Pfeiffer D, Smith RD. Principles and practical application of the receiver-operating characteristic analysis for diagnostic tests. Prev Vet Med. 2000;45(1-2):23-41. (PMID: 10802332)

25. Swets JA. Measuring the accuracy of diagnostic systems. Science. 1988;240(4857):1285-1293. (PMID: 3287615)

26. Kharroubi A, Saba E, Ghannam I, Darwish H. Evaluation of the validity of osteoporosis and fracture risk assessment tools (IOF One Minute Test, SCORE, and FRAX) in postmenopausal Palestinian women. Arch Osteoporos. 2017 Dec;12(1):6. (PMID: 28013446) 